REPRESENTATION THEORY

An Electronic Journal of the American Mathematical Society

Volume 3, Pages 354-372 (October 5, 1999)

S $1088-4165(99) 00062-\mathrm{X}$

\title{
MODULE EXTENSIONS OVER CLASSICAL LIE SUPERALGEBRAS
}

\author{
EDWARD S. LETZTER
}

\begin{abstract}
We study certain filtrations of indecomposable injective modules over classical Lie superalgebras, applying a general approach for noetherian rings developed by Brown, Jategaonkar, Lenagan, and Warfield. To indicate the consequences of our analysis, suppose that $\mathfrak{g}$ is a complex classical simple Lie superalgebra and that $E$ is an indecomposable injective $\mathfrak{g}$-module with nonzero (and so necessarily simple) socle $L$. (Recall that every essential extension of $L$, and in particular every nonsplit extension of $L$ by a simple module, can be formed from $\mathfrak{g}$-subfactors of $E$.) A direct transposition of the Lie algebra theory to this setting is impossible. However, we are able to present a finite upper bound, easily calculated and dependent only on $\mathfrak{g}$, for the number of isomorphism classes of simple highest weight $\mathfrak{g}$-modules appearing as $\mathfrak{g}$-subfactors of $E$.
\end{abstract}

\section{INTRODUCTION}

This paper is about prime ideals, indecomposable injective modules, and noetherian ring extensions-building upon [4, 12, 16, 17], [19], [32]. But our work is primarily motivated by, and applicable to, basic questions arising in the representation theory of classical Lie superalgebras.

1.1. To place our study in context, let $\mathfrak{g}$ be a complex semisimple Lie algebra with Cartan subalgebra $\mathfrak{h}$ and Weyl group $W$. It is well known, within the corresponding category $\mathcal{O}$, that the Ext (i.e., Ext ${ }^{1}$ ) groups of simple modules can be computed using Kazhdan-Lusztig polynomials $[1,7,10]$. Moreover, calculating Ext within the category of all $\mathfrak{g}$-modules, the following is elementary: Let $\lambda, \lambda^{\prime} \in \mathfrak{h}^{*}$. If $L(\lambda)$ and $L\left(\lambda^{\prime}\right)$ are simple highest weight $\mathfrak{g}$-modules for which $\operatorname{Ext}\left(L(\lambda), L\left(\lambda^{\prime}\right)\right) \neq 0$, then $\lambda$ and $\lambda^{\prime}$ occur in the same $W$-orbit in $\mathfrak{h}^{*}$. Of course, there are no nonsplit extensions of finite dimensional $\mathfrak{g}$-modules.

Generalizing further, let $E$ be the injective hull of $L(\lambda)$, and recall that every essential $\mathfrak{g}$-extension of $L(\lambda)$, and in particular every nonsplit extension of $L(\lambda)$ by a simple $\mathfrak{g}$-module, can be formed from $\mathfrak{g}$-subfactors of $E$. It follows easily, if $L\left(\lambda^{\prime}\right)$ is isomorphic to a simple subfactor of $E$, that $\lambda$ and $\lambda^{\prime}$ are contained within a single $W$-orbit; see (5.2). In comparison, if $\mathfrak{g}$ is solvable then the injective hull of a simple finite (and hence one-)dimensional $\mathfrak{g}$-module can have infinitely many pairwise nonisomorphic simple $\mathfrak{g}$-subfactors (cf. $[4, \S 6]$, recalling that windingautomorphism orbits of prime ideals in this case are either infinite or singletons).

Received by the editors November 20, 1998 and, in revised form July 14, 1999.

1991 Mathematics Subject Classification. Primary 16P40, 17A70; Secondary 17B35.

This research was partially supported by grants from the National Science Foundation.

(C)1999 American Mathematical Society 
1.2. Now, and for the remainder of this section, let $\mathfrak{g}=\mathfrak{g}_{0} \oplus \mathfrak{g}_{1}$ be a complex classical simple Lie superalgebra. (Only in this section, "g-module" will mean " $\mathbb{Z}_{2}$-graded $\mathfrak{g}$-module.") The above theory does not smoothly carry over to this setting, as is dramatically illustrated by the following example of Musson [26, §4]: Over $\operatorname{sl}(2,1)$ there exists an infinite series $L_{1}, L_{2}, \ldots$ of finite dimensional simple modules, no two of which are isomorphic, such that each $\operatorname{Ext}_{s l(2,1)}\left(L_{i}, L_{i+1}\right) \neq 0$. In particular, Kazhdan-Lusztig theory (cf. [29]) does not appear to apply. The extension theory for simple highest weight $\mathfrak{g}$-modules (in the sense, e.g., of $[25,27]$ or see (6.4)) is still largely unknown.

To demonstrate the impact of our study on these issues, let $E$ be an indecomposable injective $\mathfrak{g}$-module with nonzero socle (i.e., $E$ is the injective hull of a simple $\mathfrak{g}$-module), and let $X$ be the set of simple highest weight $\mathfrak{g}$-subfactors (up to isomorphism) of $E$. In view of Musson's example, and the Lie algebra case, it is natural to ask whether $X$ can be infinite. We calculate a finite upper boundeasily determined from $\mathfrak{g}$ and not dependent on $E$ - for the cardinality of $X$; see (6.7). More precise bounds, concerning nonsplit extensions of simple highest weight modules, are also given; see (6.5). Our bounds do not appear to be very sharp, as they significantly overestimate matters in the case when $\mathfrak{g}=\mathfrak{g}_{0}$.

To more fully describe the results of this paper, in the language of noetherian ring theory, let $\mathbf{V}$ be the enveloping algebra of $\mathfrak{g}$. The set of fundamental primes of an injective $\mathbf{V}$-module was defined in [17], following [4, 12]; see (2.6). Now let $P$ be a prime ideal of $\mathbf{V}$, and let $\operatorname{Fund}(P)$ denote the union of the sets of fundamental primes of indecomposable injective $\mathbf{V}$-modules with associated prime $P$. Our main theorem establishes a finite upper bound - easily calculated from $\mathfrak{g}$ and not dependent on $P$ - for the cardinality of Fund $(P)$; see (6.3). Sharper bounds are found for the number of prime ideals "linked" to $P$ by a nonsplit extension of prime submodules; see (2.3ii) and (6.3).

Our general approach is similar to [16], studying "lying over"-type properties of fundamental primes in ring extensions. Also, all of our results for $\mathfrak{g}$ depend on the longstanding and well-known theory for reductive Lie algebras.

\section{Noetherian modules}

All of the results discussed in this section should be considered already known, even in cases when they have not previously appeared explicitly in the literature; in such instances proofs are included for completeness. Some of the definitions introduced are nonstandard but are especially convenient for our later analysis. The reader is referred to [9] or [23] for general background information on noetherian rings.

2.1. Throughout this section, $R$ will denote a noetherian ring. The set of prime ideals of $R$ will be denoted Spec $R$ and the set of left primitive ideals of $R$ will be denoted $\operatorname{Prim} R$. The category of $R$-modules will be referred to as $\operatorname{Mod} R$, and Ext will denote Ext $^{1}$. The annihilator in $R$ of a left $R$-module $M$ will be denoted $\operatorname{ann}_{R} M$, and the annihilator in $R$ of a right $R$-module $N$ will be denoted ann $N_{R}$. Unless otherwise designated, "module" will mean "left module."

2.2. (Following [9].) Let $M$ be a nonzero $R$-module.

(i) If $Q$ is a (necessarily prime) ideal of $R$ maximal among annihilators of nonzero $R$-submodules of $M$, then ann $Q_{M}=\{m \in M \mid Q . m=0\}$ is termed an affiliated 
submodule of $M$. A series

$$
0=M_{0} \subset M_{1} \subset \cdots \subset M_{n}=M
$$

of $R$-submodules of $M$ is called an affiliated series for $M$ if each $M_{i} / M_{i-1}$ is an affiliated submodule of $M / M_{i-1}$, for $1 \leq i \leq n$; the prime ideals $\operatorname{ann}_{R}\left(M_{i} / M_{i-1}\right)$ are the affiliated primes of the series. More generally, a prime ideal of $R$ that is an affiliated prime of some affiliated series of $M$ is referred to as an affiliated prime of $M$.

(ii) If $S$ is a ring and $M$ is an $R$-S-bimodule, then the affiliated series in (i) consists of $R$-S-sub-bimodules of $M$; if $M$ has finite length as an $R$-S-bimodule, then the set of affiliated primes of $M$ (viewed as a left $R$-module) coincides with the set of left annihilators of $R$-S-bimodule composition factors of $M$.

(iii) If a prime ideal $Q$ of $R$ is the annihilator of a submodule of $M$, then $Q$ is an annihilator prime of $M$. If $Q=\operatorname{ann}_{R} M^{\prime}=\operatorname{ann}_{R} M$ for all nonzero $R$-submodules $M^{\prime}$ of $M$, and $M \neq 0$, then $M$ is said to be a prime, or more precisely, a $Q$-prime $R$-module. The annihilator of a prime module is necessarily a prime ideal, and an annihilator of a prime $R$-submodule of $M$ is termed an associated prime of $M$. Note that the annihilator of an affiliated submodule of $M$ is an associated prime. Also, every nonzero module over a noetherian ring contains a prime submodule.

(iv) If $M$ is uniform (i.e., does not contain a direct sum of two nonzero submodules), then it has exactly one associated prime ideal, which is also the unique ideal of $R$ maximal among annihilators of nonzero submodules of $M$. If $M$ has finite uniform dimension (i.e., contains no direct sum of infinitely many nonzero submodules), then $M$ can be embedded into a finite direct sum of uniform $R$-module factors of $M$ such that the associated prime of each uniform factor is an associated prime of $M$. One way to verify this last statement is to recall that the injective hull of $M$, which is an essential extension of $M$ (i.e., $M$ intersects every nonzero $R$-submodule of its injective hull nontrivially), will be a finite direct sum of uniform injective $R$-modules; see, for example, $[9$, Chapter 4] for more details. Also, recall that an injective $R$-module is indecomposable if and only if it is uniform.

\subsection{Let $Q_{\alpha}$ and $Q_{\beta}$ be prime ideals of $R$.}

(i) Let $A$ and $B$ be prime noetherian rings, and suppose that $C$ is a nonzero $A$ - $B$-bimodule finitely generated and torsion free on each side; we say that $C$ is a bond from $A$ to $B$. (A bimodule finitely generated on each side, over prime noetherian rings, is torsion free on each side if and only if it is faithful and prime on each side; see, e.g., $[12,5.1 .1]$.) It follows, for example, from $[9,7.16]$ that if $A$ is right primitive, then $B$ is right primitive, and if $B$ is left primitive, then $A$ is left primitive. Now suppose there are ideals $I \subset J$ of $R$ such that $\operatorname{ann}_{R}(I / J)=Q_{\alpha}$, such that $\operatorname{ann}(I / J)_{R}=Q_{\beta}$, and such that $I / J$ is a bond from $R / Q_{\alpha}$ to $R / Q_{\beta}$. Then we say that $I / J$ is an ideal link from $Q_{\alpha}$ to $Q_{\beta}$ and write $Q_{\alpha} \approx Q_{\beta}$. (But " $\approx$ " is not necessarily an equivalence relation.) If $Q_{\alpha} Q_{\beta} \subseteq I \subset J=Q_{\alpha} \cap Q_{\beta}$, then we say that there is a link from $Q_{\alpha}$ to $Q_{\beta}$ and write $Q_{\alpha} \rightsquigarrow Q_{\beta}$. Occasionally, for emphasis, we will refer to links as direct links. If there exist prime ideals $Q_{1}, \ldots, Q_{m}$ and a sequence of links $Q_{\alpha} \rightsquigarrow Q_{1} \rightsquigarrow \cdots \rightsquigarrow Q_{m} \rightsquigarrow Q_{\beta}$, then we write $Q_{\alpha} \rightsquigarrow \cdots \rightsquigarrow Q_{\beta}$. (When writing $Q_{\alpha} \rightsquigarrow \cdots \rightsquigarrow Q_{\beta}$, we include the possibility that $Q_{\alpha}=Q_{\beta}$ and no link exists from $Q_{\alpha}$ to itself.) In the resulting graph structure on Spec $R$, the connected component containing a prime ideal $Q$ is termed the clique of $Q$ and denoted Clique $(Q)$. 
(ii) We will say that a finitely generated uniform $R$-module $M$ is an external link from $Q_{\alpha}$ to $Q_{\beta}$, and write $Q_{\alpha} \stackrel{\text { ext }}{\rightsquigarrow} Q_{\beta}$, provided that the following condition holds: There exists a short exact sequence

$$
0 \rightarrow M_{\alpha} \rightarrow M \rightarrow M_{\beta} \rightarrow 0
$$

of uniform $R$-modules such that $0 \subset M_{\alpha} \subset M$ is an affiliated series, such that $Q_{\alpha}=\operatorname{ann}_{R} M_{\alpha}$, such that $Q_{\beta}=\operatorname{ann}_{R} M_{\beta}$, and such that $\operatorname{ann}_{R} M^{\prime}=\operatorname{ann}_{R} M$ for all $R$-submodules $M^{\prime}$ of $M$ not contained in $M_{\alpha}$. When there are prime ideals $Q_{1}, \ldots, Q_{m}$ and a sequence of external links $Q_{\alpha} \stackrel{\text { ext }}{\rightsquigarrow} Q_{1} \stackrel{\text { ext }}{\rightsquigarrow} \ldots \stackrel{\text { ext }}{\rightsquigarrow} Q_{m} \stackrel{\text { ext }}{\rightsquigarrow} Q_{\beta}$, we write $Q_{\alpha} \stackrel{\text { ext }}{\rightsquigarrow} \ldots \stackrel{\text { ext }}{\rightsquigarrow} Q_{\beta}$. (As before, $Q_{\alpha} \stackrel{\text { ext }}{\rightsquigarrow} \ldots \stackrel{\text { ext }}{\rightsquigarrow} Q_{\alpha}$ whether or not $Q_{\alpha} \stackrel{\text { ext }}{\rightsquigarrow} Q_{\alpha}$.) With respect to this graph structure on $\operatorname{Spec} R$, the connected component containing a prime ideal $Q$ is termed the external clique of $Q$ and denoted ext-Clique $(Q)$.

(iii) (Cf. $[12,6.1 .3]$.) Let $M$ be an external link from $Q_{\alpha}$ to $Q_{\beta}$, and set $I=\operatorname{ann}_{R} M$. Then Jategaonkar's Main Lemma (as stated, e.g., in [9, 11.1]) asserts that one of the following two cases must occur: (1) $Q_{\alpha} \cap Q_{\beta} / I$ is a link from $Q_{\alpha}$ to $Q_{\beta}$, or $(2) Q_{\beta}=I \subsetneq Q_{\alpha}$.

It follows from $[3,2.3 i i]$ (cf. $[9,11.2]$ ) that if $Q_{\alpha} \rightsquigarrow Q_{\beta}$, then $Q_{\alpha} \stackrel{\text { ext }}{\rightsquigarrow} Q_{\beta}$.

(iv) The condition in (ii) can be weakened somewhat. Let $N$ be a uniform $R$ module, and suppose that $Q_{\alpha}$ is the unique associated prime ideal of $N$. As noted previously, $Q_{\alpha}$ is the unique maximal annihilator in $R$ of nonzero submodules of $N$. Set $N_{\alpha}=\operatorname{ann}\left(Q_{\alpha}\right)_{N}$. Suppose further that $0 \subset N_{\alpha} \subset N$ is an affiliated series, and that $Q_{\beta}=\operatorname{ann} N / N_{\alpha}$. Setting $I$ equal to the ideal of $R$ maximal among annihilators of submodules not containing $N_{\alpha}$, we see that $M=\operatorname{ann} I_{N}$ is an external link from $Q_{\alpha}$ to $Q_{\beta}$.

(v) If for all external links between prime ideals of $R$, only case (1) in (iii) holds, then $R$ satisfies the (left) strong second layer condition.

(vi) Suppose that $R$ satisfies the strong second layer condition and that $Q_{\alpha} \approx Q_{\beta}$. Then $Q_{\alpha} \rightsquigarrow \cdots \rightsquigarrow Q_{\beta}$; see $[12,8.2 .4]$.

Although the following lemma is probably well known, we do not know of a suitable reference. The proof is adapted from $[12,7.1 .2]$, which applies to the case when $R$ satisfies the strong second layer condition.

2.4. Lemma. Let $M$ be a finitely generated nonzero $R$-module, and let $X$ denote the union of the external cliques of the associated primes of $M$. Then there exist $Q_{1}, \ldots, Q_{t} \in X$ such that $Q_{i}$ is the annihilator of a prime $R$-module subfactor of $M$, for $1 \leq i \leq t$, and such that $Q_{1} \cdots Q_{t} M=0$. If $M$ has finite length, then $\left\{Q_{1}, \ldots, Q_{t}\right\}$ can be chosen to be the set of annihilators of composition factors of $M$.

Proof. By noetherian induction, we may assume that the conclusion of the lemma holds for all of the proper $R$-module factors of $M$. Now suppose that $M$ is not uniform. As noted in (2.2iv), $M$ embeds into a finite direct sum of proper uniform $R$-module factors of $M$, each of whose associated primes is an associated prime of $M$; the lemma follows in this case. Therefore, we may assume that $M$ is uniform. Let $Q$ denote the unique associated prime of $M$, and let $N=\operatorname{ann}_{M} Q$. If $M$ has finite length, then $Q$ is the annihilator of a simple submodule of $N$. If $Q^{\prime}$ is any associated prime of $M / N$, then by (2.3iv) we can find a submodule of (the uniform module) $M$ providing an external link from $Q$ to $Q^{\prime}$. Therefore, since the conclusion of the lemma holds for $M / N$, the desired conclusion also holds for $M$. 
The following is certainly well known, but as above we do not know of an appropriate reference.

2.5. Lemma. Suppose that $Q_{\alpha}$ and $Q_{\beta}$ are prime ideals of $R$ such that $Q_{\alpha} \stackrel{\text { ext }}{\rightsquigarrow} Q_{\beta}$. Then $Q_{\alpha} \cap Z=Q_{\beta} \cap Z$, where $Z$ is the center of $R$.

Proof. Let $M$ be an external link from $Q_{\alpha}$ to $Q_{\beta}$, as in (2.3ii). When $Q_{\alpha} \rightsquigarrow Q_{\beta}$, the conclusion of the lemma follows immediately. Therefore, we may assume without loss of generality that $Q_{\alpha} \supsetneq Q_{\beta}=\operatorname{ann}_{R} M$, as in (2.3iii), and we may further assume without loss that $Q_{\beta}=0$. (Note, however, that the image in $R / Q_{\beta}$ of the original $Z$ may be smaller than the center of $R / Q_{\beta}$.) Now let $I=Q_{\alpha} \cap Z$, and set $J=R I$. By [9, 11.13], for example, $J$ is an AR-ideal (see, e.g., [9, p. 190]). Thus $J^{n} M=0$ for some positive integer $n$, by [9, 11.11b], since $J$ annihilates a nonzero submodule of $M$, and since $M$ is uniform. Hence $I^{n} M=0$. But $M$ is a faithful $Z$-module, and $Z$ is prime. Consequently, $I=J=0$, and the lemma follows.

2.6. We now briefly review the construction and basic properties of the fundamental series of an $R$-module, following [17] (cf. [4], [12, Chapter 9]). To start, assume that $R$ is an algebra of finite Gelfand-Kirillov dimension (GK-dimension) over a field $k$ (cf. [15] or [23] for definitions and background), that the GK-dimension of every $R$-module with finite GK-dimension is an integer, and that if $0 \rightarrow L \rightarrow M \rightarrow N \rightarrow 0$ is an exact sequence of $R$-modules, then the GK-dimension of $M$ is equal to the maximum of the GK-dimensions of $L$ and $N$. (The preceding assumptions are summarized by saying that $R$ has exact integer $G K$-dimension.)

(i) Let $M$ be a nonzero $R$-module. Say that $M$ is $(\alpha$-) annihilator homogeneous for some integer $\alpha$ if $\operatorname{GKdim}\left(R / \operatorname{ann}_{R} N\right)=\alpha$ for all nonzero finitely generated submodules $N$ of $M$. If $X$ is a set of semiprime ideals of $R$, then the intersection of the ideals in any nonempty subset of $X$ is referred to as an $X$-semiprime ideal of $R$.

(ii) Assume that $M$ is an annihilator homogeneous nonzero $R$-module, and set $F_{0}(M)=0$. For $j=1,2, \ldots$, set $X_{j}(M)$ equal to the set of associated primes of $M / F_{j-1}(M)$, and set

$$
F_{j}(M)=\left\{m \in M \mid I m \in F_{j-1}(M) \text { for some } X_{j}(M) \text {-semiprime ideal } I \text { of } R\right\} \text {. }
$$

The series $F_{0}(M) \subset F_{1}(M) \subset \cdots$ is the fundamental series for $M$. In $[17,3.2]$ it is shown that $M=\bigcup_{j} F_{j}(M)$. The set of fundamental primes of $M$, denoted Fund $(M)$, is defined as $\bigcup_{j} X_{j}(M)$.

(iii) Now let $M$ be an arbitrary (i.e., not necessarily annihilator homogeneous) nonzero $R$-module, and let $\alpha_{1}<\alpha_{2}<\cdots<\alpha_{n}$ be the distinct integers arising as the GK-dimensions of $R / \operatorname{ann}_{R} N$, for finitely generated submodules $N$ of $M$. Set $G_{0}(M)=0$, and for $1 \leq i \leq n$ set $G_{i}(M)$ equal to

$\left\{m \in M \mid m \in G_{i-1}(M)\right.$ or $\left.\operatorname{GKdim}\left(R / \operatorname{ann}_{R}\left[R m+G_{i-1}(M) / G_{i-1}(M)\right]\right)=\alpha_{i}\right\}$. In $[17,4.2]$ it is proved that

$$
G_{0}(M) \subsetneq G_{1}(M) \subsetneq \cdots \subsetneq G_{n}(M)=M
$$

and that each $G_{i}(M) / G_{i-1}(M)$ is an $\alpha_{i}$-annihilator homogeneous $R$-module. Set

$$
\operatorname{Fund}_{i}(M)=\operatorname{Fund}\left(G_{i}(M) / G_{i-1}(M)\right),
$$

and define the fundamental primes of $M$, denoted Fund $(M)$, to be $\bigcup_{i} \operatorname{Fund}_{i}(M)$. 
(iv) Continue to let $M$ be an arbitrary nonzero $R$-module, and let $Q$ be an annihilator prime of $M$. In [17,4.4] it is proved that there exist submodules $H$ and $J$ of $M$ such that $Q H=0$ and such that $H / J$ is $Q$-prime. Choosing $h \in H \backslash J$, we see that $Q=\operatorname{ann}_{R} R$.h. Consequently, the set of annihilator primes of $M$ is equal to the set of prime annihilators of finitely generated submodules of $M$.

2.7. (i) Let $A$ and $B$ denote algebras of finite GK-dimension, and let $C$ denote an $A$-B-bimodule finitely generated on each side. Then $\operatorname{GKdim}\left(A / \operatorname{ann}_{A} C\right)=$ $\operatorname{GKdim}\left({ }_{A} C\right)=\operatorname{GKdim}\left(C_{B}\right)=\operatorname{GKdim}\left(B / \operatorname{ann} C_{B}\right)$; see, for example, [15, 5.3]. Consequently, if $A$ is a subalgebra of $B$, and if $B$ is finitely generated on either the right or left as an $A$-module, then $\operatorname{GKdim}(A)=\operatorname{GKdim}(B)$.

(ii) Suppose that $R$ has finite GK-dimension. Let $Q_{\alpha}$ and $Q_{\beta}$ be prime ideals of $R$. If $Q_{\alpha} \subseteq Q_{\beta}$, then $Q_{\alpha}=Q_{\beta}$ if and only if $\operatorname{GKdim}\left(R / Q_{\alpha}\right)=\operatorname{GKdim}\left(R / Q_{\beta}\right)$; see $[15,3.16]$.

2.8. Assume that $R$ has exact integer GK-dimension. Let $E$ be an injective $R$ module, and let $Q_{\beta}$ be a prime ideal of $R$. It is proved in $[17,5.4]$ (noting (2.7i)) that $Q_{\beta}$ is a fundamental prime of $E$ if and only if there exists an annihilator prime $Q_{\alpha}$ of $E$, and an ideal link $I / J$ from $Q_{\alpha}$ to $Q_{\beta}$, such that $\operatorname{GKdim}\left(R / Q_{\alpha}\right)=$ $\operatorname{GKdim}(R / J)=\operatorname{GKdim}\left(R / Q_{\beta}\right)$.

2.9. Suppose that $R$ has finite GK-dimension. Let $Q_{\alpha}$ and $Q_{\beta}$ be prime ideals of $R$.

(i) In view of (2.8), we will say that there is a strong ideal link from $Q_{\alpha}$ to $Q_{\beta}$ if there is an ideal link $I / J$ such that $\operatorname{GKdim}\left(R / Q_{\alpha}\right)=\operatorname{GKdim}(R / J)=$ $\operatorname{GKdim}\left(R / Q_{\beta}\right)$.

(ii) Suppose that there is an ideal link $I / J$ from $Q_{\alpha}$ to $Q_{\beta}$ and that $\operatorname{GKdim}\left(R / Q_{\alpha}\right)$ $=\operatorname{GKdim}\left(R / Q_{\beta}\right)=\operatorname{GKdim}(R)$. Since $J$ is contained in both $Q_{\alpha}$ and $Q_{\beta}$, we see that $\operatorname{GKdim}(R) \geq \operatorname{GKdim}(R / J) \geq \operatorname{GKdim}\left(R / Q_{\alpha}\right)=\operatorname{GKdim}(R)$. Therefore, $I / J$ is a strong ideal link.

(iii) Now assume that $R$ has exact integer GK-dimension. It follows from the remarks after $[17,5.4]$, when there exists a strong ideal link from $Q_{\alpha}$ to $Q_{\beta}$, that $Q_{\alpha} \rightsquigarrow \cdots \rightsquigarrow Q_{\beta}$. Hence if $E$ is an injective $R$-module, and $Q_{\beta}$ is a fundamental prime of $E$, then there exists an annihilator prime $Q_{\alpha}$ of $E$ such that $Q_{\alpha} \rightsquigarrow \cdots \rightsquigarrow Q_{\beta}$.

2.10. Assume that $R$ has exact integer GK-dimension, and suppose that $\left(Q_{\alpha} \cap Q_{\beta}\right) / J$ is a link from $Q_{\alpha}$ to $Q_{\beta}$. It follows from (2.7i) and, for example, $[15,5.7]$ that $\operatorname{GKdim}\left(R / Q_{\alpha}\right)=\operatorname{GKdim}(R / J)=\operatorname{GKdim}\left(R / Q_{\beta}\right)$. We see in this case that a direct link is a strong ideal link.

2.11. Lemma. Assume that $R$ has exact integer GK-dimension, that $M$ is a uniform R-module, that $Q_{\alpha}$ is the unique associated prime of $M$, and that $Q_{\beta}$ is an annihilator prime of $M$. Then $Q_{\alpha} \stackrel{\text { ext }}{\rightsquigarrow} \stackrel{\text { ext }}{\rightsquigarrow Q_{\beta}}$.

Proof. By (2.6iv), $Q_{\beta}$ is the annihilator of a finitely generated submodule of $M$; this submodule is uniform and its unique associated prime is $Q_{\alpha}$. Therefore, we can reduce to the case where $Q_{\beta}=0$ and where $M$ is a finitely generated faithful $R$-module. However, by (2.4), there exist prime ideals $Q_{1}, \ldots, Q_{t}$ in the external clique of $Q_{\alpha}$ such that $Q_{1} \cdots Q_{t} M=0$. Thus $Q_{1} \cdots Q_{t}=0$, and so $Q_{i}=0$ for some $1 \leq i \leq t$. The lemma follows. 
2.12. Continue to assume that $R$ has exact integer GK-dimension, and let $Q$ be a prime ideal of $R$.

(i) Define Fund $(Q)$, the set of fundamental primes of $Q$, to be the union of the sets of fundamental primes of indecomposable (equivalently, uniform) injective $R$ modules for which $Q$ is the unique associated prime. It follows from (2.9iii) and (2.11) that $\operatorname{Fund}(Q) \subseteq$ ext-Clique $(Q)$.

(ii) If $M$ is a finite length uniform $R$-module with associated primitive ideal $Q$, then it is not hard to verify that the annihilators of the composition factors of $M$ are all contained in $\operatorname{Fund}(Q)$.

(iii) Suppose that $M$ is a uniform $R$-module with associated prime $Q$, that $L$ is an arbitrary simple $R$-module subfactor of $M$, and that $\operatorname{ann}_{R} L=Q_{\beta}$. We may embed $M$ into a uniform injective $R$-module $E$, and the filtration of $E$ described in (2.6) is exhaustive. Hence there exists $Q_{\alpha} \in \operatorname{Fund}(Q) \subseteq$ ext-Clique $(Q)$ such that $Q_{\alpha} \subseteq Q_{\beta}$.

\section{NOETHERIAN SUPERALGEBRAS}

We now briefly review some generalities, relevant to the study of Lie superaglebras, from the theory of $\mathbb{Z}_{2}$-graded rings.

3.1. (i) Throughout this section, $k$ will denote a field not of characteristic two, and $R$ will denote a noetherian $k$-algebra. We will further assume, throughout, that $R=R_{0} \oplus R_{1}$ is $\mathbb{Z}_{2}$-graded (i.e., $R$ is an associative superalgebra).

(i) Henceforth, we will use the term graded to mean " $\mathbb{Z}_{2}$-graded" and "homogeneous with respect to the $\mathbb{Z}_{2}$-grading." If $X=X_{0} \oplus X_{1}$ is a $\left(\mathbb{Z}_{2}\right.$-)graded $k$-vector space, then the elements of $X_{0}$ will be called even and the elements of $X_{1}$ will be called odd. Degree-preserving linear maps between graded $k$-vector spaces will be termed graded. However, a $k$-vector space should be assumed graded only when explicitly specified. Ungraded will mean "not necessarily graded."

(ii) Let $X=X_{0} \oplus X_{1}$ be a graded $k$-vector space. There is an automorphism $\sigma$ of $X$, obviously of order two, mapping each even element to itself and each odd element to its additive inverse. Also, $X_{0}$ is identical to $X^{\sigma}$, the subspace of $\sigma$-invariants. Now let $Y$ be an ungraded subspace of $X$. The unique maximum graded subspace of $X$ contained in $Y$ will be termed $\operatorname{gr} Y$, and it is easy to see that $Y$ is graded if and only if $\sigma(Y)=Y$. Therefore, $\operatorname{gr} Y=Y \cap \sigma(Y)$.

(iii) Applied to $R, \sigma$ is a $k$-algebra automorphism. Therefore, $R_{0}$ is noetherian and $R$ is finitely generated on each side as an $R_{0}$-module [24, 1.12, 5.9]. Applied to a graded $R$-module, $\sigma$ permutes the ungraded submodules.

(iv) The category of graded left $R$-modules - with graded morphisms - will be denoted Grmod $R$.

3.2. (i) We will say that a graded $R$-module $M$ is graded-simple provided the only nonzero graded submodule of $M$ is itself (i.e., $M$ is a simple object in Grmod $R$ ), a condition that occurs if and only if $M_{0}$ and $M_{1}$ are simple $R_{0}$-modules. Consequently, a graded $R$-module $N$ has finite length as an ungraded $R$-module if and and only if $N$ has finite length in Grmod $R$.

(ii) The annihilators of graded-simple $R$-modules are the (left) graded-primitive ideals, and the set of graded-primitive ideals of $R$ will be denoted $\operatorname{GrPrim} R$.

(iii) Assume that $M$ is a graded-simple $R$-module with annihilator $p$. The $R_{0^{-}}$ module structure of $M$ ensures that its length as an ungraded $R$-module is no 
greater than two. Moreover, if $N$ is an ungraded simple $R$-submodule of $M$, and $\operatorname{ann}_{R} N=P$, then $M=N+\sigma(N)$, and $p=\operatorname{gr}(P)=P \cap \sigma(P)$.

(iv) Let $L$ be a maximal ungraded left ideal of $R$. Set $P=\operatorname{ann}_{R}(R / L)$, and set $M=R / L \cap \sigma(L)$. Then $M \subseteq(R / L) \oplus(R / \sigma(L))$ has length no greater than two, as either an ungraded or graded $R$-module, and $p=\operatorname{gr}(P)=\operatorname{ann}_{R} M$. It is now straightforward to check that $p$ is graded-primitive.

(v) We see from (iii) and (iv) that a graded ideal $p$ of $R$ is graded-primitive if and only if there exists a primitive ideal $P$ of $R$ for which $p=\operatorname{gr}(P)=P \cap \sigma(P)$.

3.3. (i) A graded ideal of $R$ is graded-prime when it contains no product of strictly larger graded ideals, and a graded ideal is graded-semiprime when it is the intersection of graded-prime ideals. Also, $R$ will be termed graded-prime (resp. gradedsemiprime) when the zero ideal of $R$ is graded-prime (resp. graded-semiprime). It is proved as follows that an ideal $p$ of $R$ is graded-prime if and only if there exists a prime ideal $P$ of $R$ such that $p=\operatorname{gr}(P)=P \cap \sigma(P)$ : First, when $P$ is a prime ideal of $R$, it is straightforward to check that $\operatorname{gr}(P)$ is graded-prime. Conversely, let $p$ be a graded prime ideal of $R$. Since $R$ is noetherian there exist prime ideals $P_{1}, \ldots, P_{t}$ of $R$, each containing $p$, such that $P_{1} \cdots P_{t} \subseteq p$. But $\operatorname{gr}\left(P_{1}\right) \cdots \operatorname{gr}\left(P_{t}\right) \subseteq p$, and each of the $\operatorname{gr}\left(P_{i}\right)$ contains $p$. Thus $p=\operatorname{gr}\left(P_{i}\right)$ for some $1 \leq i \leq t$.

(ii) If $P$ is a prime ideal of $R$, then $P \cap R_{0}=\sigma(P) \cap R_{0}=\operatorname{gr}(P) \cap R_{0}=P^{\sigma}$ is a semiprime ideal of $R_{0}$; see, for example, [24, 1.5].

(iii) Suppose that $R$ is graded-semiprime. Then $R_{0}$ is semiprime by (ii). Let $X$ denote the Ore set of regular elements of $R_{0}$. By $[24,5.3], X$ is an Ore set of regular elements of $R$, and the left Ore localization $X^{-1} R$ is equal to the Goldie quotient ring of $R$. Because $X$ consists only of even elements of $R$, the grading on $R$ extends naturally to a grading of $X^{-1} R$.

Now let $M$ be a graded $R$-module, and let $Y$ denote the Ore set of regular elements of $R$. Because $X^{-1} R$ is the Goldie quotient ring of $R$, a submodule $N$ of $M$ is $X$-torsion (i.e., torsion with respect to $X$ ) if and only if $N$ is torsion (i.e., $Y$-torsion). Since $X$ consists only of even elements, the torsion submodule of $M$ is therefore graded. Also, $M$ is torsion free if and only if $M$ is $X$-torsion free.

(iv) Continue to assume that $R$ is graded-semiprime. Let $M=M_{0} \oplus M_{1}$ be a graded $R$ - $R$-bimodule (i.e., $R_{i} M_{j}$ and $M_{j} R_{i}$ are both contained in $M_{i+j}$ ). By (iii), the left torsion submodule $T$ of $M$ is a graded bimodule, and by [12, 5.1.1], $T$ is the largest left unfaithful graded $R$ - $R$-sub-bimodule of $M$. We may therefore define a graded-bond between graded-prime rings $A$ and $B$ to be a graded bimodule finitely generated and torsion free on each side. Graded-ideal-links and graded(-direct-)links can be similarly defined.

(v) Suppose that $R$ is graded-prime. Then its Goldie quotient ring is a gradedsimple (i.e., containing no proper graded ideals) Artinian ring. Now suppose that $A$ is a graded-simple artinian $\left(\mathbb{Z}_{2}\right.$ ) graded ring. By (i), $A$ is either simple artinian or equal to a direct product of two isomorphic simple artinian rings. In the first case, $A$ is an $m \times m$ matrix ring over a division ring and we say that $m$ is the graded-rank of $A$. In the second case, $A$ is a direct product of two $n \times n$ matrix rings over division rings, and we say that $n$ is the graded-rank of $A$. The graded-Goldie-rank of $R$ is the graded-rank of its Goldie quotient ring.

3.4. Assume that $R$ has exact integer GK-dimension (see (2.6)) and that $M$ is a graded $R$-module. 
(i) Let $I$ be an ideal of $R$. Since $R$ has exact integer GK-dimension, $\operatorname{GKdim}(R / I)$ $=\operatorname{GKdim}(R / \sigma(I))=\operatorname{GKdim}[R /(I \cap \sigma(I))]=\operatorname{GKdim}(R / \operatorname{gr}(I))$, by [15, 5.7]. If $m \in M$ and $I=\operatorname{ann}_{R} R m$, then $\sigma(I)=\operatorname{ann}_{R} R \sigma(m)$.

(ii) Let $\alpha$ be the least positive integer occurring among $\operatorname{GKdim}_{R}(N)$ for finitely generated nonzero $R$-submodules $N$ of $M$. It follows from (i) that $G(M)=$ $\left\{m \in M \mid \operatorname{GKdim}\left(R / \operatorname{ann}_{R} R m\right)=\alpha\right\}$ is a $\sigma$-stable $R$-submodule of $M$, and so $G(M)$ is a graded submodule of $M$. In particular, $G(M)$ is equal to the sum of homogeneous $m \in M$ such that $\operatorname{GKdim}\left(R / \operatorname{ann}_{R} R m\right)=\alpha$.

(iii) Let $\alpha_{1}<\alpha_{2}<\cdots<\alpha_{n}$ be the integers occurring among $\operatorname{GKdim}\left(R / \operatorname{ann}_{R} N\right)$ for finitely generated nonzero submodules $N$ of $M$. By (ii), each of the submodules in the series $0=G_{0}(M) \subset G_{1}(M) \subset \cdots \subset G_{n}(M)=M$, as defined in (2.6iii), is graded.

(iv) $\operatorname{Suppose}$ that $\operatorname{GKdim}\left(R / \operatorname{ann}_{R} L\right)=\alpha$ for all finitely generated nonzero graded $R$-submodules $L$ of $M$; we will say in this case that $M$ is $(\alpha$-)gradedannihilator-homogenous. Let $N$ be a finitely generated nonzero ungraded submodule of $M$, and suppose that $\operatorname{ann}_{R} N=I$. Then $\alpha=\operatorname{GKdim}[R / \operatorname{ann}(N+\sigma(N))]=$ $\operatorname{GKdim}[R / \operatorname{gr}(I)]=\operatorname{GKdim}(R / I)$, by (i). Hence $M$ is $\alpha$-annihilator homogeneous (see $(2.6 \mathrm{i}))$.

(v) We see from (iv) that a graded $R$-module is $\alpha$-graded-annihilator homogeneous if and only if it is $\alpha$-annihilator homogeneous. For $1 \leq i \leq n$, the graded subfactors $G_{i}(M) / G_{i-1}(M)$ of (iii) are $\alpha_{i}$-graded-annihilator-homogeneous, by (2.6iii).

3.5. The following duality principle of Cohen and Montgomery [5] allows us to apply ungraded noetherian ring theory to $\operatorname{Grmod} R$. Set $\widehat{R}=R \# k\left[\mathbb{Z}_{2}\right]^{*}$ as in $[5, \S 1]$. When viewed as either a left or right module over its $k$-subalgebra $R, \widehat{R}$ is free of rank two. Hence $\widehat{R}$ is noetherian, and if $R$ has finite GK-dimension, then so does $\widehat{R}$. Furthermore, if $R$ has exact integer GK-dimension, then so does $\widehat{R}$, by $[22,1.6]$.

In $[5,2.2]$ it is proved that the categories $\operatorname{Grmod} R$ and $\operatorname{Mod} \widehat{R}$ are isomorphic.

3.6. (i) In view of (3.2)-(3.5), we now claim that the results mentioned in $\S 2$, and their prerequisites, are valid if the modules and bimodules involved are replaced with the corresponding graded objects. (The terms "dimension over $k$ " and "GKdimension" retain their usual ungraded definitions even when applied to graded modules.) A formal proof of this assertion will be omitted.

(ii) We will continue to use the prefix graded- to denote the graded analogue of an ungraded term.

(iii) The existence of a graded-external-link from a graded-prime ideal $p_{\alpha}$ to a graded-prime ideal $p_{\beta}$ will be denoted $p_{\alpha} \stackrel{\text { ext }}{\rightsquigarrow} p_{\beta}$, and a graded-link from $p_{\alpha}$ to $p_{\beta}$ will be denoted $p_{\alpha} \rightsquigarrow p_{\beta}$. When $R$ has exact integer GK-dimension, $\operatorname{GrFund}(M)$ will refer to the fundamental graded-primes of a graded $R$-module $M$, and if $p$ is a graded-prime ideal of $R$ we will use $\operatorname{GrFund}(p)$ to denote the union of the sets of the fundamental graded-primes of graded-uniform graded-injective $R$-modules.

(iv) Note that the graded-injective hull of a graded $R$-module $M$ will be a maximal graded-essential extension of $M$.

\section{EXTENSIONS OF RINGS}

This section is devoted to developing some preparatory results concerning ring extensions finitely generated on one side. 
4.1. Throughout this section $S$ will denote a noetherian algebra of finite GKdimension over a field $k$, and $R$ will denote a noetherian subalgebra of $S$ such that $S=s_{1} R+\cdots+s_{m} R$ for some $s_{1}, \ldots, s_{m} \in S$. Further suppose, throughout, that $P$ is a prime ideal of $S$ and that $Q$ is a prime ideal of $R$.

(i) Suppose that $Q$ is minimal over $P \cap R$; we say that $P$ lies over $Q$ and that $Q$ lies under $P$. Since $R$ is noetherian, at least one and at most finitely many prime ideals of $R$ lie under $P$. It follows from $[18,1.1]$, for example, that there is a bond from $S / P$ to $R / Q$. Consequently, by $(2.7 \mathrm{i}), \operatorname{GKdim}(R / Q)=\operatorname{GKdim}(S / P)$. Of course, it is easy to see that $P$ has finite codimension (in $S$ ) if and only if $Q$ has finite codimension (in $R$ ). From (2.3i) it follows that if $P$ is right primitive, then $Q$ is right primitive, and if $Q$ is left primitive, then $P$ is left primitive.

(ii) If $P$ lies over $Q$, and if $Q$ is a left annihilator prime of $S / P$, then we say that $P$ lies directly over $Q$ and that $Q$ lies directly under $P$. (Cf. [8, 5.1].) There exists at least one prime ideal of $R$ lying directly under $P$ (see, e.g., $[21,2.6 \mathrm{i}]$ ).

4.2. Let $F$ denote the Goldie quotient ring of $R / Q$. Because $S$ is finitely generated as a right $R$-module, $S \otimes_{R} F$ has finite length as a right $F$-module. It is proved in $[21,2.4]$ that $P$ lies directly over $Q$ if and only if $P$ is the left annihilator in $S$ of an irreducible $S$ - $F$-bimodule factor of $S \otimes_{R} F$.

The proof of the following relies on the Joseph-Small Additivity Principle (cf. $[2,13,31])$.

4.3. Proposition. (i) $P$ lies directly over at most $m$ prime ideals of $R$. (ii) $Q$ lies directly under at most $m$ prime ideals of $S$.

Proof. (i) Let $Q_{1}, \ldots, Q_{s}$ be prime ideals of $R$ lying directly under $P$. For $1 \leq i \leq s$, let $F_{i}$ denote the Goldie quotient ring of $R / Q_{i}$. It follows from (4.2) that $P$ is the left annihilator of an irreducible $S$ - $F_{i}$-bimodule factor of $S \otimes_{R} F_{i}$. By [9, 7.23], a version of the Additivity Principle,

$$
\operatorname{rank}(S / P) \leq \operatorname{length}\left(S \otimes_{R} F_{i}\right)_{F_{i}} \leq m \cdot \operatorname{rank}\left(R / Q_{i}\right),
$$

where $\operatorname{rank}()$ denotes Goldie rank and length ()$_{F_{i}}$ denotes composition length as a right $F_{i}$-module. However, it now follows from the standard version of the Additivity Principle (see, e.g., $[23,4.5 .4])$ that

$$
\begin{aligned}
& \operatorname{rank}\left(R / Q_{1}\right)+\cdots+\operatorname{rank}\left(R / Q_{s}\right) \leq \operatorname{rank}(S / P) \\
& \leq m \cdot \min \left\{\operatorname{rank}\left(R / Q_{1}\right), \ldots, \operatorname{rank}\left(R / Q_{s}\right)\right\} .
\end{aligned}
$$

Hence $s \leq m$.

(ii) Suppose that $P_{1}, \ldots, P_{t}$ are prime ideals of $S$ lying directly over $Q$. Another application of the Additivity Principle (this time see, e.g., $[9,7.26]$ ) ensures that $\operatorname{rank}(R / Q) \leq \operatorname{rank}\left(S / P_{j}\right)$, for $1 \leq j \leq t$. Let $F$ denote the Goldie quotient ring of $R / Q$; it follows from (4.2) that $P_{j}$ is the annihilator of an irreducible $S$ - $F$-bimodule factor of $S \otimes_{R} F$. Therefore, again using [9, 7.23], we see that

$$
\operatorname{rank}\left(S / P_{1}\right)+\cdots+\operatorname{rank}\left(S / P_{t}\right) \leq m \cdot \min \left\{\operatorname{rank}\left(S / P_{1}\right), \ldots, \operatorname{rank}\left(S / P_{t}\right)\right\},
$$

and so $t \leq m$.

The following is analogous to $[20,3.4]$.

4.4. Proposition. Suppose that $P$ lies over $Q$. Then there exists a prime ideal $Q^{\prime}$ of $R$ such that $Q^{\prime}$ lies directly under $P$ and such that there is a strong ideal link from $Q^{\prime}$ to $Q$. 
Proof. We may assume, without loss of generality, that $P=0$. We first find a prime ideal $Q^{\prime}$ of $R$ such that $Q^{\prime}$ is an annihilator prime of $S$ and such that there is an ideal link from $Q^{\prime}$ to $Q$. Let $X$ denote the set of elements of $R$ regular modulo its nilradical. Because $S$ has finite GK-dimension and is finitely generated as a right $R$-module, it follows from $[2,2.2]$ that $X$ consists of regular elements of $S$ and is a right Ore set in both $R$ and $S$. Consequently, the embedding of $R$ into $S$ extends to an embedding of the right quotient ring $R X^{-1}$ into $S X^{-1}$. Note that $R X^{-1}$ is artinian by Small's Theorem (see, e.g., $[9,10.9]$ ), that $S X^{-1}$ is finitely generated as a right $R X^{-1}$-module, and that $S X^{-1}$ is the Goldie quotient ring of $S$. Set $A=R X^{-1}, B=S X^{-1}$, and $K=Q X^{-1}$.

As noted in (2.2iv), we can embed $A$ (as a left $A$-module) into a finite direct sum of uniform left $A$-module factors of $A$ such that the unique associated prime of each uniform factor is an associated prime of $A$; let $L_{1}, \ldots, L_{s}$ denote these prime ideals. By $[16,2.3]$, there now exist prime ideals $K_{1}, \ldots, K_{t}$ such that $0=$ $K_{1} K_{2} \cdots K_{t} A=K_{1} K_{2} \cdots K_{t}$ and such that for each $1 \leq j \leq t$ there exists $1 \leq i \leq s$ for which $L_{i} \approx K_{j}$. However, since $K_{1} \cdots K_{t} \subseteq K$, and since $K$ is a minimal prime ideal of $A$, it follows that $K=K_{j}$ for some $1 \leq j \leq t$. In particular, we have found an annihilator prime $L$, of the left $A$-module $B$, such that $L \approx K$. Setting $Q^{\prime}=L \cap R$, we see that $Q^{\prime}$ is an annihilator prime of $R$ for which there exists an ideal link from $Q^{\prime}$ to $Q$.

By $(2.7 \mathrm{i})$ and (4.1i), $\operatorname{GKdim}(R)=\operatorname{GKdim}(S)=\operatorname{GKdim}(R / Q)=\operatorname{GKdim}\left(R / Q^{\prime}\right)$. Hence there exists a strong ideal link from $Q^{\prime}$ to $Q$, by (2.9ii). The lemma follows.

The next lemma is another consequence of the Additivity Principle.

4.5. Lemma. Assume that $S$ is prime.

(i) Let $I$ be an ideal of $R$ not contained within any minimal prime ideal of $R$. Then there exists a nonzero ideal of $S$ contained within $S I$. If $S$ is finitely generated as a left $R$-module, then there exists a nonzero ideal of $S$ contained within IS.

(ii) Let $M$ be a faithful prime $S$-module, and let $I$ be an ideal of $R$ such that $I N=0$ for some nonzero $R$-submodule $N$ of $M$. Then $I$ is contained within a minimal prime ideal of $R$. Consequently, if the prime ideal $Q$ of $R$ is an annihilator prime of $M$ (viewed as an $R$-module), then $Q$ is a minimal prime ideal of $R$.

Proof. (i) See [21, 2.1].

(ii) If $I$ is not contained within any minimal prime ideal of $R$, then, by (i), there exists a nonzero ideal $J$ of $S$ such that $J(S N)=J N \subseteq S I N=0$, a contradiction to the choice of $M$.

The following is similar to $[19,2.5]$.

4.6. Lemma. Assume that $S$ is prime and finitely generated as both a left and right $R$-module. Let $M$ be a finitely generated, uniform, faithful $S$-module, and assume that $P_{\alpha}$ is the unique associated prime of $M$. Then there exist prime ideals $Q_{\alpha}$ and $Q_{\beta}$ of $R$ such that $P_{\alpha}$ lies over $Q_{\alpha}$, such that $Q_{\beta}$ is a minimal prime ideal of $R$, and such that $Q_{\alpha} \stackrel{e x t}{\rightsquigarrow \rightsquigarrow} \stackrel{\text { ext }}{\rightsquigarrow Q_{\beta}}$.

Proof. As observed in (2.2iv), $P_{\alpha}$ is the unique maximal annihilator, in $S$, of nonzero $S$-submodules of $M$. Set $N=\operatorname{ann}\left(P_{\alpha}\right)_{M}$. It follows from (4.5ii) that if $Q$ is an annihilator prime of $N$, viewed as a left $R$-module, then $P_{\alpha}$ lies over $Q$. Next, choose $L$ to be an $R$-submodule of $M$ maximal such that $L \cap N=0$, and set 
$K=M / L$. The $R$-module embedding $N \hookrightarrow K$ is essential, forcing every associated prime, in $R$, of $K$ to also be an associated prime of $N$. In particular, $P_{\alpha}$ lies over each associated prime of $K$. Now let $X$ denote the union of the external cliques of the associated primes of $K$. By (2.4), there exist $Q_{1}, \ldots, Q_{t} \in X$ such that $Q_{1} \cdots Q_{t} K=0$. Set $I=Q_{1} \cdots Q_{t}$. Then $I S M=I M \subseteq L$.

Suppose that $I$ is not contained within a minimal prime ideal of $R$. Then by (4.5i), there exists a nonzero ideal $J$ of $S$ contained within $I S$. Note that $J M$ is nonzero because $M$ is faithful, and that $J M$ is then an $S$-submodule of $M$ contained in $L$. Hence $J M \cap N=0$, contradicting the uniformity of $M$ as an $S$-module. Thus $I$ is contained in a minimal prime ideal of $R$, and one of the $Q_{1}, \ldots, Q_{t}$ must be a minimal prime ideal of $R$. The lemma follows.

4.7. Lemma. Suppose that $S$ has exact integer $G K$-dimension and that $S$ is finitely generated as both a left and right $R$-module. Let $M$ be a uniform $S$-module. Let $P_{\alpha}$ be the unique associated prime, in $S$, of $M$, and suppose that $P_{\beta}$ is an annihilator prime, in $S$, of $M$. Then there exist prime ideals $Q_{\alpha}$ and $Q_{\beta}$ of $R$ such that $Q_{\alpha}$ lies under $P_{\alpha}$, such that $Q_{\beta}$ lies under $P_{\beta}$, and such that $Q_{\alpha} \stackrel{\text { ext }}{\rightsquigarrow} \stackrel{\text { ext }}{\rightsquigarrow} Q_{\beta}$.

Proof. First, we may assume without loss of generality that $P_{\beta}=\operatorname{ann}_{S} M$. Therefore, we can reduce to the case where $P_{\beta}=0$ and $M$ is faithful as an $S$-module. By (2.6iv), we may further assume that $M$ is finitely generated as an $S$-module. The lemma now follows from (4.6).

4.8. Proposition. Let $T$ be a noetherian k-algebra, containing $S$ as a subalgebra, such that $T=t_{1} S+\cdots+t_{\ell} S$. Let $w$ be a positive integer. Assume there exist, for every prime ideal $Q_{\alpha}$ of $R$, at most $w$ prime ideals $Q_{\beta}$ such that either $Q_{\alpha}$ is strongly ideal linked to $Q_{\beta}$ or $Q_{\beta}$ is strongly ideal linked to $Q_{\alpha}$. Let $J$ be a prime ideal of $T$.

(i) There exist at most mw prime ideals of $S$ lying over $Q$.

(ii) There exist at most $m w$ prime ideals of $R$ lying under $P$.

(iii) There exist at most $\ell m w$ prime ideals of $T$ lying over $P$.

(iv) There exist at most $\ell m^{2} w$ prime ideals of $S$ lying under $J$.

Proof. Parts (i) and (ii) follow from (4.3) and (4.4).

(iii) Suppose that $J$ lies over $P$ and that $P$ lies over $Q$. Then $J \cap R \subseteq Q$. It follows from (4.1i) that the GK-dimensions of $T / J, S / P$, and $R / Q$ all coincide. Therefore, $Q$ must be minimal over $J \cap R$, by (2.7ii), and so $J$ lies over $Q$. It follows from (i) that at most $\ell m w$ prime ideals of $T$ can lie over $Q$, and (iii) follows.

(iv) Suppose that $P$ lies under $J$ and directly over $Q$; as noted in (4.1ii), $P$ lies directly over at least one prime ideal of $R$. As in the proof of (iii), $Q$ lies under $J$. By (ii), there are no more than $\ell m w$ prime ideals of $R$ lying under $J$. By (4.3i), there are at most $m$ prime ideals of $S$ lying directly over $Q$. Part (iv) follows.

The following is similar, for example, to $[32,6.6]$.

4.9. Lemma. Let $I / J$ be a strong ideal link from the prime ideal $Q_{\alpha}$ to the prime ideal $Q_{\beta}$ of $R$. Further suppose that $S J \cap R=J$ (e.g., suppose that $S$ is free as a right $R$-module). Then there exist prime ideals $P_{\alpha}$ and $P_{\beta}$ of $S$ such that $P_{\alpha}$ lies over $Q_{\alpha}$, such that $P_{\beta}$ lies over $Q_{\beta}$, and such there is a strong ideal link from $P_{\alpha}$ to $P_{\beta}$. 
Proof. Note that $\operatorname{ann}(S / S J)_{R}=J$, and let $K=\operatorname{ann}_{S}(S / S J)$. By assumption, $K \cap R \subseteq J$, and so we may suppose without loss of generality that $K=0$. By (2.7i) and the assumption that $I / J$ is a strong ideal link,

$$
\operatorname{GKdim}(R)=\operatorname{GKdim}(S)=\operatorname{GKdim}(R / J)=\operatorname{GKdim}\left(R / Q_{\alpha}\right)=\operatorname{GKdim}\left(R / Q_{\beta}\right) \text {. }
$$

Since $\operatorname{GKdim}\left(R / Q_{\alpha}\right)=\operatorname{GKdim}\left(R / Q_{\beta}\right)=\operatorname{GKdim}(R)$, we see from (2.7ii) that $Q_{\alpha}$ and $Q_{\beta}$ are minimal prime ideals of $R$. It now follows from $[32,6.3 \mathrm{i}]$ that there exist prime ideals $P_{\alpha}$ and $P_{\beta}$ of $S$ such that $P_{\alpha}$ lies over $Q_{\alpha}$, such that $P_{\beta}$ lies over $Q_{\beta}$, and such that there is an ideal link $L / M$ from $P_{\alpha}$ to $P_{\beta}$. Since $\operatorname{GKdim}\left(S / P_{\alpha}\right)=\operatorname{GKdim}\left(S / P_{\beta}\right)=\operatorname{GKdim}\left(R / Q_{\beta}\right)=\operatorname{GKdim}(S)$, it follows from (2.9ii) that there is a strong ideal link from $P_{\alpha}$ to $P_{\beta}$.

\section{EXtensions OF ENVELOPING ALGEBRAS}

In this section we establish bounds on the sizes of sets of fundamental primes, in a setting that includes finite extensions of enveloping algebras of reductive Lie algebras.

5.1. Let $k$ be a field, and let $R$ be a noetherian $k$-algebra of exact integer GKdimension (see (2.6)). Throughout this section, $S$ will denote a (necessarily noetherian) $k$-algebra, containing $R$ as a subalgebra, such that $S$ is free of rank $\ell$ as a left $R$-module and is generated as a right $R$-module by no more than $m$ elements. Further assume, throughout, that every external clique (see (2.3ii)) of prime ideals of $R$ has cardinality no greater than some fixed positive integer $w$.

5.2. For a general example fitting the description of $R$ in (5.1), suppose that $k$ is an algebraically closed field of characteristic zero, that $\mathfrak{a}$ is a (finite dimensional) reductive $k$-Lie algebra, and that $W$ is the associated Weyl group. Recall that the enveloping algebra of a finite dimensional Lie algebra is noetherian.

(i) Setting $U=U(\mathfrak{a})$, and $Z$ equal to the center of $U$, it follows from [30, Theorem 1] that the sets of prime ideals of $U$ having common intersection with $Z$ correspond exactly with suitably defined $W$-orbits in the prime spectrum of the symmetric algebra $S\left(\mathfrak{h}^{*}\right)$. In particular, by (2.5), the external cliques in Spec $U$ have cardinality no greater than $|W|$.

(ii) It is shown, for example, in [15, Chapter 6] that enveloping algebras of finite dimensional Lie algebras, over any field, have exact integer GK-dimension. So suppose, for the moment, that $R$ is the enveloping algebra of a finite dimensional Lie algebra. Recall, if $Q$ is a prime ideal of $R$, that $Q$ is left primitive if and only if it is right primitive. It follows, when $P$ is a prime ideal of $S$, that $P$ is left primitive if and only if it is right primitive (see, e.g., [18]).

(iii) Recall that the (left, or equivalently, right) primitive ideals of $U$ (as defined in (i)) are precisely the prime ideals intersecting to maximal ideals of $Z$, that the minimal primitive ideals of $U$ are the ideals of the form $U \chi$ for maximal ideals $\chi$ of $Z$, and that the set of annihilators of Verma modules over $U$ coincides with the set of minimal primitive ideals of $U$. The Verma modules are uniform $U$-modules of finite length, and from Duflo's Theorem it follows that every primitive ideal is the annihilator of a simple factor of a Verma module. (See [6] or [11] for details.) It now follows from (2.4) that the external clique in $\operatorname{Spec} U$ of a primitive ideal $P$ of $U$ is exactly the set of primitive ideals whose intersection with $Z$ equals $P \cap Z$.

(iv) It follows from (iii) that if $Q_{\alpha}$ and $Q_{\beta}$ are primitive ideals of $U$ such that $Q_{\alpha} \subseteq Q_{\beta}$, then $Q_{\alpha}$ and $Q_{\beta}$ are in the same external clique. Suppose that $M$ is a 
uniform $U$-module with associated prime $Q$, that $Q$ is primitive, and that $L$ is a simple $U$-module factor of $M$. It now follows from (2.12iii) that the annihilator in $U$ of $L$ is in the external clique of $Q$.

5.3. Let $T=M_{\ell}(R)$. By $[22,1.6]$, both $S$ and $T$ have exact integer GK-dimension. In particular, any pair of strongly ideal linked prime ideals of $R, S$, or $T$ is contained within a common clique, by (2.9iii). As noted in [19, 7.1], there is a $k$-algebra embedding of $S$ into $T$ such that $T$ is a free right $S$-module of rank $\ell$. Of course, every external clique of prime ideals of $T$ has cardinality no greater than $w$.

5.4. Lemma. Let $P$ be a prime ideal of $S$.

(i) Let $C$ denote the union of the external cliques of the prime ideals of $R$ lying under $P$, and let $C^{\prime}$ denote the set of prime ideals in $S$ lying over ideals in $C$. Then $\left|C^{\prime}\right| \leq m^{2} w$.

(ii) Let $D$ denote the union of the external cliques of the prime ideals of $T$ lying over $P$, and let $D^{\prime}$ denote the set of prime ideals in $S$ lying under ideals in $D$. Then $\left|D^{\prime}\right| \leq \ell^{2} m^{3} w^{3}$.

Proof. (i) By (4.3ii) and (4.4), $|C| \leq m w$. By (4.4), $C^{\prime}$ is equal to the set of prime ideals of $S$ lying directly over ideals in $C$, and so from (4.3i) it follows that $\left|C^{\prime}\right| \leq m^{2} w$.

(ii) By (4.8iii), $|D| \leq \ell m w^{2}$, and so it follows from (4.8iv) that $\left|D^{\prime}\right| \leq \ell^{2} m^{3} w^{3}$.

Fundamental series and primes (cf. $[4,12,17])$ are discussed in (2.6) and (2.12).

5.5. Theorem. If $P$ is a prime ideal of $S$, then $|\operatorname{Fund}(P)| \leq \ell^{2} m^{5} w^{4}$.

Proof. First, let $A$ denote the set of annihilator primes of uniform $S$-modules with associated prime $P$. Set $C$ equal to the union of the external cliques of prime ideals of $R$ lying under $P$, and let $C^{\prime}$ denote the set of prime ideals of $S$ lying over ideals in $C$. By (4.7), $A \subseteq C^{\prime}$, and so $|A| \leq m^{2} w$, by (5.4i).

Second, let $P_{\alpha}$ be a prime ideal of $S$, and let $B$ denote the set of prime ideals $P_{\beta}$ of $S$ for which there is a strong ideal link from $P_{\alpha}$ to $P_{\beta}$. Let $D$ be the union of the external cliques of the prime ideals of $T$ lying over $P_{\alpha}$, and let $D^{\prime}$ denote the set of prime ideals of $S$ lying under prime ideals in $D$. By (2.12i) and (4.9), $B \subseteq D^{\prime}$. Thus $|B| \leq \ell^{2} m^{3} w^{3}$, by (5.4ii).

It follows from (2.8) that $|\operatorname{Fund}(P)| \leq|A||B|$. The theorem follows.

5.6. A bound similar to, and sharper than, that of (5.5) can be established for external links (see (2.3)) and can in turn be applied to extensions of simple modules, as follows: Suppose that $0 \rightarrow L_{\alpha} \rightarrow M \rightarrow L_{\beta} \rightarrow 0$ is a nonsplit extension of simple modules, that $P_{\alpha}=\operatorname{ann}_{S} L_{\alpha}$, and that $P_{\beta}=\operatorname{ann}_{S} L_{\beta}$; then $M$ is an external link from $P_{\alpha}$ to $P_{\beta}$.

5.7. Theorem. Let $P$ be a prime ideal of $S$.

(i) There exist at most $\ell^{2} m^{3} w^{3}$ prime ideals $P^{\prime}$ of $S$ such that $P \rightsquigarrow P^{\prime}$.

(ii) There exist at most $m^{2} w$ prime ideals $P^{\prime}$ of $S$ such that $P \stackrel{\text { ext }}{\rightsquigarrow} P^{\prime}$ but $P \leadsto P^{\prime}$.

(iii) There exist at most $m^{2} w+\ell^{2} m^{3} w^{3}$ prime ideals $P^{\prime}$ of $S$ such that $P \stackrel{\text { ext }}{\rightsquigarrow} P^{\prime}$.

Proof. (i) By (2.10) and (4.9), if $P \rightsquigarrow P^{\prime}$ then there exist prime ideals $J$ and $J^{\prime}$ of $T$ such that $J$ lies over $P$, such that $J^{\prime}$ lies over $P^{\prime}$, and such that there is a strong ideal link from $J$ to $J^{\prime}$. Part (i) now follows from (2.12i) and (5.4ii). 
(ii) Let $M$ be an external link from $P$ to $P^{\prime}$, and suppose that $P \leftrightarrow P^{\prime}$. By (2.3iii), $P^{\prime}=\operatorname{ann}_{S} M$, and so by (4.6), or (4.7), there exist prime ideals $Q$ and $Q^{\prime}$ of $R$ such that $P$ lies over $Q, P^{\prime}$ lies over $Q^{\prime}$, and $Q \stackrel{\text { ext }}{\rightsquigarrow} \stackrel{\text { ext }}{\rightsquigarrow} Q^{\prime}$. Part (ii) now follows from (5.4i).

(iii) This part now follows from (i), (ii), and (2.3iii).

5.8. Assume that $R=U(\mathfrak{a})$, for $\mathfrak{a}$ as in (5.2).

(i) Distinct primitive ideals with finite codimension in $R$ cannot have equal intersection with its center, and the analogous statement also holds true for $T$. Hence, there are no ideal links in either $R$ or $T$ between distinct finite codimensional primitive ideals. It now follows, for example, from (4.9) that linked, primitive, finite codimensional ideals in $S$ lie under a common finite codimensional primitive ideal of $T$. Moreover, there is a nonsplit extension of finite dimensional simple $S$-modules if and only if there exists a direct link between their corresponding annihilators.

(ii) Suppose that $J$ is a finite codimensional primitive ideal of $T$, that $P$ is a finite codimensional primitive ideal of $S$, and that $Q$ is finite codimensional primitive ideal of $R$. By (i) and (4.4), $Q$ lies under $J$ (resp. $P$ ) if and only if $Q$ lies directly under $J$ (resp. $P$ ). Therefore, by (4.3ii) there exist at most $\ell m$ prime ideals of $R$ lying under $J$, and by (4.3i) there exist at most $m$ prime ideals of $S$ lying over $Q$. Thus, as in the proof of (4.8), there exist at most $\ell \mathrm{m}^{2}$ prime ideals of $S$ lying under $J$.

(iii) Let $L$ be an arbitrary finite dimensional simple $S$-module, and let $X$ denote the set of finite dimensional simple $S$-modules $L^{\prime}$ for which either $\operatorname{Ext}_{S}\left(L, L^{\prime}\right) \neq 0$ or $\operatorname{Ext}_{S}\left(L^{\prime}, L\right) \neq 0$. It follows from (i) and (ii) that $|X| \leq \ell m^{2}$.

(iv) Let $P_{\alpha}$ and $P_{\beta}$ be prime ideals of $S$, and suppose that $M$ is an external link from $P_{\alpha}$ to $P_{\beta}$. We can show as follows that $P_{\alpha}$ is primitive if and only if $P_{\beta}$ is primitive. First, if $P_{\alpha} \rightsquigarrow P_{\beta}$, then $P_{\alpha}$ is primitive if and only if $P_{\beta}$ is primitive, by (2.3i) and (5.2ii). We may therefore assume that $P_{\alpha} \uparrow_{\rightarrow} P_{\beta}$. By (2.3iii) $P_{\beta}=\operatorname{ann}_{S} M$, and by (4.7) it follows that there exist prime ideals $Q_{\alpha}$ and $Q_{\beta}$ of $R$ such that $P_{\alpha}$ lies over $Q_{\alpha}$, such that $P_{\beta}$ lies over $Q_{\beta}$, and such that $Q_{\alpha} \stackrel{\text { ext }}{\rightsquigarrow} \stackrel{\text { ext }}{\rightsquigarrow} Q_{\beta}$. It now follows from (5.2iii) and (4.1i) that $P_{\alpha}$ is primitive if and only if $P_{\beta}$ is primitive.

5.9. Retaining the assumptions of (5.1), suppose further that the characteristic of $k$ is not equal to two, that $S$ is $\left(\mathbb{Z}_{2^{-}}\right)$graded, that $R=R_{0}$ is a trivially graded subalgebra of $S_{0}$, and that the free left $R$-module basis for $S$ is comprised of homogeneous elements. The algebra $T$ is then also graded, and $R$ and $S$ are graded subalgebras of $T$. Following (3.6), the results in this section remain valid when the modules and bimodules involved are replaced with their graded counterparts.

\section{Classical Lie superalgebras}

We now apply the results of the preceding sections to the representation theory of Lie superalgebras. The reader is referred to [14] or [28] for background information.

6.1. (i) Let $k$ be an algebraically closed field of characteristic zero. Assume throughout this section that $\mathfrak{g}=\mathfrak{g}_{0} \oplus \mathfrak{g}_{1}$ is a finite dimensional $k$-Lie superalgebra, that $\mathbf{V}$ is the associative enveloping algebra of $\mathfrak{g}$, and that $\mathbf{U}$ is the enveloping algebra of the finite dimensional Lie algebra $\mathfrak{g}_{0}$.

(ii) It follows from the Poincare-Birkhoff-Witt Theorem for Lie superalgebras that $\mathbf{V}$ contains $\mathbf{U}$ as a subalgebra and that $\mathbf{V}$ is free of rank $2^{\operatorname{dim} g_{1}}$ as both a right 
and left $\mathbf{U}$-module (and that the right and left bases can be chosen to coincide). Hence $\mathbf{V}$ is noetherian. As remarked in (5.3), $\mathbf{U}$ and $\mathbf{V}$ have exact integer GKdimension.

(iii) The $\left(\mathbb{Z}_{2^{-}}\right)$grading on $\mathfrak{g}$ extends to a grading of $\mathbf{V}$, and $\mathbf{U} \subseteq \mathbf{V}_{0}$. Furthermore, the free left and right common $\mathbf{U}$-basis for $\mathbf{V}$ can be chosen to consist of homogeneous elements. In particular, the remarks in (5.9) apply.

6.2. (i) It follows from (5.2ii) that a prime ideal of $\mathbf{V}$ is left primitive if and only if it is right primitive.

(ii) Assume that $\mathfrak{g}$ is simple (i.e., it contains no nonzero graded ideals other than itself). If $\mathfrak{g}_{1}$ is completely reducible as an ad $\mathfrak{g}_{0}$-module - a condition that occurs if and only if $\mathfrak{g}_{0}$ is reductive (see, e.g., [28, Theorem 1, p. 101]) — then $\mathfrak{g}$ is called classical.

(iii) Suppose that $\mathfrak{g}_{0}$ is reductive (with $\mathfrak{g}$ not necessarily simple), and let $P$ be a (left or right) primitive ideal of $\mathbf{V}$. It follows from (5.8iv) that the external clique, in Spec $\mathbf{V}$, of $P$ consists only of primitive ideals, and so it follows from (2.12i) that the prime ideals in $\operatorname{Fund}(P)$ are all primitive. Similarly, if $p$ is a graded-primitive ideal of $\mathbf{V}$, then the graded-prime ideals in $\operatorname{GrFund}(p)$ are graded-primitive.

The next theorem summarizes the immediate consequences of (5.2), (5.5), (5.7iii), and (5.8iii). Passage to the graded case follows from (3.6) and (5.9).

6.3. Theorem. Assume that $\mathfrak{g}_{0}$ is reductive (e.g., $\mathfrak{g}$ is classical simple) and that the Weyl group associated to $\mathfrak{g}_{0}$ has cardinality $w$. Set $\ell=2^{\operatorname{dim}}$. Let $P$ be a prime ideal of $\mathbf{V}$, and let $p$ be a graded-prime ideal of $\mathbf{V}$.

(i) $|\operatorname{Fund}(P)| \leq \ell^{7} w^{4}$.

(i') $|\operatorname{GrFund}(p)| \leq \ell^{7} w^{4}$.

(ii) There exist at most $\ell^{2} w+\ell^{5} w^{3}$ prime ideals $P^{\prime}$ of $\mathbf{V}$ such that $P \stackrel{\text { ext }}{\rightsquigarrow P^{\prime}}$.

(ii') There exist at most $\ell^{2} w+\ell^{5} w^{3}$ graded-prime ideals $p^{\prime}$ of $\mathbf{V}$ such that $p \stackrel{\text { ext }}{p^{\prime}}$.

(iii) Let $L$ be a simple finite dimensional $\mathbf{V}$-module. Then there are at most $\ell^{3}$ finite dimensional simple $\mathbf{V}$-modules $L^{\prime}$ for which $\operatorname{Ext}\left(L, L^{\prime}\right)$ or $\operatorname{Ext}\left(L^{\prime}, L\right)$, calculated in $\operatorname{Mod} \mathbf{V}$, is not equal to zero.

(iii') Let $L$ be a graded-simple finite dimensional $\mathbf{V}$-module. Then there are at most $\ell^{3}$ finite dimensional graded-simple $\mathbf{V}$-modules $L^{\prime}$ for which $\operatorname{Ext}\left(L, L^{\prime}\right)$ or $\operatorname{Ext}\left(L^{\prime}, L\right)$, calculated in $\operatorname{Grmod} \mathbf{V}$, is not equal to zero.

6.4. We now turn to highest weight modules, following [25] (cf. [27]). Assume that $\mathfrak{g}=\mathfrak{g}_{0} \oplus \mathfrak{g}_{1}$ is classical simple, that $W$ is the Weyl group of $\mathfrak{g}_{0}$, that $w=|W|$, and that $\ell=2^{\operatorname{dim} \mathfrak{g}_{1}}$.

(i) To start, fix a triangular decomposition $\mathfrak{g}=\mathfrak{n}^{-} \oplus \mathfrak{h} \oplus \mathfrak{n}^{+}$as in $[25,1.1]$. Then $\mathfrak{g}_{0}=\mathfrak{n}_{0}^{-} \oplus \mathfrak{h}_{0} \oplus \mathfrak{n}_{0}^{+}$is a triangular decomposition of $\mathfrak{g}_{0}$.

(ii) Set $\mathfrak{b}=\mathfrak{h} \oplus \mathfrak{n}^{+}$. For each $\lambda \in \mathfrak{h}_{0}^{*}$ there is a unique finite dimensional gradedsimple $\mathfrak{b}$-module $K_{\lambda}$ such that $\mathfrak{n}^{+} . K_{\lambda}=0$, and such that $h . x=\lambda(h) . x$ for all $h \in \mathfrak{h}_{0}$ and $x \in K_{\lambda}$. Moreover, all of the finite dimensional graded-simple $\mathfrak{b}$-modules are obtained, up to isomorphism, in this fashion. (See [14, §5.2], [25, §1.1].)

(iii) Fix $\lambda \in \mathfrak{h}_{0}^{*}$, and set $\widetilde{M}(\lambda)=\mathbf{V} \otimes_{U(\mathfrak{b})} K_{\lambda}$. Then $\widetilde{M}(\lambda)$ has finite length as a $\mathbf{V}$-module and has a unique maximal graded $\mathbf{V}$-submodule; the resulting gradedsimple factor module is denoted $\widetilde{L}(\lambda)$.

(iv) Extending Duflo's Theorem (see, e.g., $[11,7.4])$, Musson proved that the map $\mathfrak{h}_{0}^{*} \rightarrow \operatorname{GrPrim} \mathbf{V}$, sending $\lambda \mapsto \operatorname{ann}_{\mathbf{V}} \widetilde{L}(\lambda)$, is surjective [25, §2]. Moreover, the fibers of the preceding map all have cardinality no greater than $\ell w$; see $[21,3.2]$. 
In view of (5.6), the next proposition follows immediately from $\left(6.3 \mathrm{ii}^{\prime}\right)$ and (6.4iv).

6.5. Proposition. Retain the notation and assumptions of (6.4). Let $\lambda \in \mathfrak{h}_{0}^{*}$. Then there are at most $\ell^{3} w^{2}+\ell^{6} w^{4}$ linear forms $\lambda^{\prime} \in \mathfrak{h}_{0}^{*}$ for which

$$
\operatorname{Ext}\left(\widetilde{L}(\lambda), \widetilde{L}\left(\lambda^{\prime}\right)\right) \quad \text { or } \quad \operatorname{Ext}\left(\widetilde{L}\left(\lambda^{\prime}\right), \widetilde{L}(\lambda)\right)
$$

calculated in Grmod V, is not equal to zero.

We conclude our study by considering graded-essential extensions of gradedsimple highest weight modules.

6.6. Lemma. Retain the notation and assumptions of (6.4), and let $P$ be a primitive ideal of $\mathbf{V}$. Then there exist at most $\ell^{2} w^{3}$ primitive ideals $P^{\prime}$ of $\mathbf{V}$ such that $P \subseteq P^{\prime}$. If $p$ is a graded-primitive ideal of $\mathbf{V}$ then there exist at most $\ell^{2} w^{3}$ graded-primitive ideals $p^{\prime}$ of $\mathbf{V}$ such that $p \subseteq p^{\prime}$.

Proof. Suppose that $Q_{1}, \ldots, Q_{t}$ are the primitive (see (4.1i)) ideals of $\mathbf{U}$ lying under $P$. By (4.8ii), $t \leq \ell w$. Now suppose that $P^{\prime}$ is a primitive ideal of $\mathbf{V}$ containing $P$, and suppose that $P$ lies over the primitive ideal $Q^{\prime}$ of $\mathbf{U}$. Then $Q^{\prime}$ contains $Q_{i}$, for some $1 \leq i \leq t$, since there will be a power of $Q_{1} \cdots Q_{t}$ contained inside $P \cap \mathbf{U} \subseteq P^{\prime} \cap \mathbf{U} \subseteq Q^{\prime}$. However, there are at most $w$ primitive ideals of $\mathbf{U}$ containing $Q_{i}$, and at most $\ell w$ prime ideals of $\mathbf{V}$ lying over $Q^{\prime}$, by (4.8i). The ungraded part of the lemma now follows, and the graded case is proved similarly.

6.7. Theorem. Retain the notation and assumptions of (6.4). Let $M$ be a gradeduniform $\mathbf{V}$-module whose unique associated graded-prime ideal is graded-primitive (e.g., let $M$ be a graded-indecomposable graded-injective $\mathbf{V}$-module with a nonzero graded-socle). Then there exist at most $\ell^{10} w^{8}$ linear forms $\lambda \in \mathfrak{h}_{0}^{*}$ such that $\widetilde{L}(\lambda)$ is isomorphic to a graded-simple $\mathbf{V}$-module subfactor of $M$.

Proof. Suppose that $\widetilde{L}(\lambda)$ is a graded-simple $\mathfrak{g}$-subfactor of $M$, for some $\lambda \in \mathfrak{h}_{0}^{*}$, and set $p_{\lambda}=\operatorname{ann}_{\mathbf{V}} \widetilde{L}(\lambda)$. By (2.12iii), $p_{\lambda}$ contains some graded-prime ideal $p \in$ $G=\operatorname{GrFund}(M)$. By $\left(6.3 \mathrm{i}^{\prime}\right),|G| \leq \ell^{7} w^{4}$. But $p$ is graded-primitive, by (6.2iii), and so there exist at most $\ell^{2} w^{3}$ graded-primitive ideals of $\mathbf{V}$ containing $p$, by (6.6). However, as noted in (6.4iv), there exist at most $\ell w$ graded-simple heighest weight modules with a given graded-primitive annihilator in V. The theorem follows.

6.8. Remark. (i) I do not know the sharpness, in general, of the various bounds established in this section. Of course, when $\mathfrak{g}=\mathfrak{g}_{0}$ they significantly overestimate the situation.

(ii) Musson's example $[26, \S 4]$, described in $(1.2)$, shows that $\mathbf{V}$ may have infinite cliques (see (2.3)) of finite codimensional graded-primitive ideals.

\section{ACKNOWLEDGMENTS}

This work was inspired by questions raised by Ian Musson - posed to me in 1991. Much of the material in $\S 2$ is folklore, mostly taught to me by Tom Lenagan and Bob Warfield when I was a graduate student at the University of Washington. 


\section{REFERENCES}

1. D. Barbasch, Filtrations on Verma modules, Ann. Scient. École Norm. Sup. 16 (1983), 489494. MR 85j: 17013

2. W. Borho, Invariant dimension and restricted extension of noetherian rings, Séminaire d'Algébre Paul Dubreil et Marie-Paule Malliavin (M.-P. Malliavin, ed.), Lecture Notes in Mathematics 924, Springer, Berlin, 1982, pp. 51-71. MR 83h:16018

3. K. A. Brown, Ore sets in noetherian rings, Séminaire d'Algébre Paul Dubreil et Marie-Paule Malliavin (M.-P. Malliavin, ed.), Lecture Notes in Mathematics 1146, Springer, Berlin, 1985, pp. 355-366. MR 88b:16006

4. K. A. Brown and R. B. Warfield, Jr., The influence of ideal structure on representation theory, J. Alg. 116 (1988), 294-315. MR 89k:16026

5. M. Cohen and S. Montgomery,, Group-graded rings, smash products, and group actions, Trans. Amer. Math. Soc. 282 (1984), 237-257. MR 85i:16002

6. J. Dixmier, Enveloping Algebras: The 1996 Printing of the 1977 English Translation, Graduate Studies in Mathematics 11, American Mathematical Society, Providence, 1996. MR 97c: 17010

7. O. Gabber and A. Joseph, Towards the Kazhdan-Lusztig conjecture, Ann. Sci. École Norm. Sup. 14 (1981), 261-302. MR 83e:17009

8. K. R. Goodearl and E. S. Letzter, Prime ideals in skew and q-skew polynomial rings, Mem. Amer. Math. Soc. 109 (1994). MR 94j:16051

9. K. R. Goodearl and R. B. Warfield, Jr., An introduction to noncommutative noetherian rings, London Mathematical Society Student Texts, 16, Cambridge, New York, 1989. MR 91c:16001

10. R. S. Irving, The socle filtration of a Verma module, Ann. Sci. École Norm. Sup. 21 (1988), 47-65. MR 89h:17015

11. J. C. Jantzen, Einhullende Algebren Halbeinfacher Lie-Algebren, Ergebnisse der Mathematik, 3. Folge, Band 3, Springer, New York, 1983. MR 86c:17011

12. A. V. Jategaonkar, Localization in noetherian rings, London Mathematical Society Lecture Note Series 98, Cambridge University Press, Cambridge, 1986. MR 88c: 16005

13. A. Joseph and L. W. Small, An additivity principle for Goldie rank, Israel J. Math. 31 (1978), 105-114. MR 80j:17005

14. V. G. Kac, Lie Superalgebras, Adv. Math. 26 (1977), 8-96. MR 58:5803

15. G. R. Krause and T. H. Lenagan, Growth of algebras and Gelfand-Kirillov dimension, Pitman, London, 1985. MR 86g:16001

16. T. H. Lenagan and E. S. Letzter, The fundamental prime ideals of a noetherian prime PI ring, Proc. Edinburgh Math. Soc. 33 (1990), 113-121. MR 91b:16026

17. T. H. Lenagan and R. B. Warfield, Jr., Affiliated series and extensions of modules, J. Alg. 142 (1991), 164-187. MR 92m:16037

18. E. S. Letzter, Primitive ideals in finite extensions of noetherian rings, J. London Math. Soc. (2) 39 (1989), 427-435. MR 90f:16013

19. __ Prime ideals in finite extensions of noetherian Rings, J. Alg. 135 (1990), 412-439. MR 91m: 16020

20. Soc. 116 (1992), 645-652. MR 93a:16003

21. _ A Aijection of primitive spectra for classical Lie superalgebras of type I, J. London Math. Soc. (2) 52 (1996), 39-49. MR 96k:17016

22. M. Lorenz, On Gelfand-Kirillov dimension and related topics, J. Alg. 118 (1988), 423-437. MR 89m:16004

23. J. C. McConnell and J. C. Robson, Noncommutative noetherian rings, John Wiley and Sons, Chichester, 1987. MR 89j:16023

24. S. Montgomery, Fixed rings of finite automorphism groups of associative rings, Lecture Notes in Mathematics, no. 818, Springer, Berlin, 1980. MR 81j:16041

25. I. M. Musson, A classification of primitive ideals in the enveloping algebra of a classical Lie superalgebra, Adv. Math. 91 (1992), 252-268. MR 93c:17022

26. _ Primitive ideals in the enveloping algebra of the Lie superalgebra $\mathrm{sl}(2,1)$, J. Algebra 159 (1993), 306-331. MR 94g:17016

27. I. Penkov and V. Serganova, Generic irreducible representations of finite-dimensional Lie superalgebras, Int. J. Math. 5 (1994), 389-419. MR 95c: 17015 
28. M. Scheunert, The theory of Lie superalgebras, Lecture Notes in Mathematics, 716, Springer, Berlin, 1979. MR 80i:17005

29. V. Serganova, Kazhdan-Lusztig polynomials and character formula for the Lie superalgebra $\mathfrak{g} l(m \mid n)$, Selecta Math. (N.S.) 2 (1996), 607-651. MR 98f:17007

30. W. Soergel, The prime spectrum of the enveloping algebra of a reductive Lie algebra, Math. Z. 204 (1990), 559-581. MR 91d:17015

31. R. B. Warfield, Jr., Prime ideals in ring extensions, J. London Math. Soc. (2) 28 (1983), 453-460. MR 85e:16006

32. _ Noetherian ring extensions with trace conditions, Trans. Amer. Math. Soc. 331 (1992), 449-463. MR 92g:16032

Department of Mathematics, Texas A\&M University, College Station, Texas 77843

E-mail address: letzter@math.tamu.edu 